\title{
DETERMINACIÓN DE PARENTESCO EN LARVAS DE Pseudoplatystoma fasciatum (Linnaeus, 1766) PRODUCIDAS EN CAUTIVERIO
}

\author{
Diana CASTRO-RUIZ ${ }^{1}$, Sophie QUEROUIL ${ }^{2}$, Etienne BARAS ${ }^{2}$, Werner CHOTA-MACUYAMA ${ }^{1}$, Fabrice \\ DUPONCHELLE ${ }^{2}$, Jesús NUÑEZ ${ }^{2}$, Jean-François RENNO², Carmen GARCÍA-DÁVILA ${ }^{1}$ \\ 1 Instituto de Investigaciones de la Amazonía Peruana-IIAP, Laboratorio de Biología y Genética Molecular (LBGM). \\ Apartado 784. Iquitos, Perú. \\ 2 Institut de Recherche pour le Développement-IRD. 175 Caviar, BP 5095, 34196 Montpellier cedex 05, France.
}

\section{RESUMEN}

Un set de seis loci microsatélites fue utilizado como herramienta molecular para identificar el genotipo de los reproductores y asignar el parentesco a diferentes familias de Pseudoplatystoma fasciatum. Las progenies cultivadas en situación comunal fueron obtenidas por la reproducción inducida de los óvulos de una hembra con un pool de esperma de cuatro machos. Todos los loci microsatélites mostraron apropiada amplificación y polimorfismo, con alelos diagnósticos o combinación de alelos para identificar a los reproductores y asignar el parentesco sin riesgo a equivocarse. Los alelos nulos encontrados en los loci Pcor 7 y Pcor 8, no dificultaron la identificación de la progenie. Se obtuvieron cuatro familias de medio-hermanos (en cada etapa de muestreo). Resultados de este estudio demuestran la utilidad de las herramientas genéticas en la asignación de parentesco en familias de Pseudoplatystoma fasciatum cultivadas comunalmente.

PALABRAS CLAVES: Pseudoplatystoma fasciatum, piscicultura, microsatélites, genotipo, parentesco.

\section{PARENTAGE ASSIGNMENT IN LARVAL Pseudoplatystoma fasciatum (Linnaeus, 1766) PRODUCED IN CAPTIVITY}

\begin{abstract}
A set of six microsatellite loci was used to identify the genotype of the breeder and assign kinship in different families of Pseudoplatystoma fasciatum. The progenies bred communally were obtained by combining the eggs of a female with a pool of sperm from four males. All microsatellite loci showed appropriate amplification and polymorphism with unique alleles or a combination of alleles to identify the breeder and assign kinship without risk of error. Null alleles were encountered at loci Pcor 7 and Pcor 8, but there was no difficulty in identifying progeny. Four half-sibling groups were tested (at each stage of sampling). Results of this study demonstrate the utility of genetic tools in assigning parentage in families of Pseudoplatystoma fasciatum bred communally.
\end{abstract}

KEYWORDS: Pseudoplatystoma fasciatum, fish culture, microsatellite, genotyping, parentage. 


\section{INTRODUCCIÓN}

La doncella, Pseudoplatystoma fasciatum, es uno de los bagres amazónicos que sustenta la pesquería comercial en la región Loreto. Esta especie presenta un gran potencial para la producción comercial en toda América del Sur (Kossowski, 1996; Goulding, 1980; Reid, 1983). Debido a la calidad de su filete y su agradable sabor, genera una gran demanda en el mercado regional e internacional, que por ahora, es cubierta a través de la pesca comercial en ambientes naturales (Tello \& García, 2009). Esta demanda en los últimos años viene ocasionando el aumento de la presión de pesca, y la captura de individuos en tallas por debajo del tamaño de primera madurez sexual, lo que estaría causando la disminución de sus poblaciones naturales y pérdidas económicas para el futuro del sector pesquero (Tello \& García, 2009).

La piscicultura de esta especie, además de ser una alternativa económica que genera ingresos para la región Loreto (García et al., 2009), también es una buena opción para reducir la sobrepesca de las poblaciones naturales, contribuyendo de esta manera a la conservación del recurso (García et al., 2009). En ambientes controlados, P. fasciatum tiene una elevada tasa de crecimiento $(1,2 \mathrm{~kg}$ y $45 \mathrm{~cm}$ en 18 meses) y fecundidad (150 000 óvulos $/ \mathrm{kg}$ ). Además, de una buena resistencia a la manipulación y adaptación a dietas balanceadas (Nuñez, 2009). A pesar de estas bondades, hasta el momento la piscicultura de la doncella no ha alcanzado el desarrollo esperado. La principal limitante es la elevada tasa de mortalidad, ocasionada por el alto nivel de canibalismo que presentan sus larvas (Montalvan, 2008; Nuñez et al., 2008, Nuñez et al., 2011). El monitoreo de los progenitores y de sus progenies permite un desarrollo controlado del proceso de domesticación. El uso de los marcadores moleculares como barcode permite este mismo monitoreo en situación de cautiverio comunal de las larvas y de los alevines.

Los perfiles genéticos de DNA han sido propuestos como una herramienta para el monitoreo de la progenie de peces cultivados para la acuicultura (Harris et al., 1991). De todas las herramientas moleculares, los microsatélites (secuencias de DNA nuclear constituidas por repeticiones en tándem de entre uno a seis nucleótidos) mostraron ser las mas útiles en estudios de parentesco (Borrell et al., 2002; Castro et al., 2006; Herlin et al., 2008; Chakraborty et al., 1988; Kellogg et al., 1995; Garber et al., 2010) $\mathrm{y}$ en la reconstrucción de pedigree de poblaciones comúnmente cultivadas para la acuicultura (Harris et al., 1991), debido a que presentaron una gran variabilidad en peces marinos y de agua dulce (De Woody \& Avise, 2000; Revaldaves et al., 2005;
Pereira et al., 2009). Por lo tanto, hacen posible la realización de estudios que involucran el cultivo de diferentes progenies en ambientes comunales (estudios que antes fueron imposibles, debido a problemas relacionados con la determinación del parentesco). Los perfiles de microsatélites hacen posible la asignación de individuos a grupos de familias cultivadas en un mismo tanque, minimizando así la variación ambiental y permitiendo evaluar la relación intra e inter-familiar. En este trabajo se pretende identificar el genotipo de los reproductores y establecer el parentesco a un grupo de larvas de $P$. fasciatum cultivadas en situación comunal, provenientes de la fertilización de una hembra con cuatro machos.

\section{MATERIALES Y MÉTODOS}

La investigación fue realizada en los laboratorios de reproducción inducida de Peces y de Biología y Genética Molecular (LBGM), del centro experimental de Quistococha - Instituto de Investigaciones de la Amazonía Peruana (IIAP), Ubicado en el 4,5 km de la Carretera Iquitos-Nauta, Distrito de San Juan Bautista, Provincia de Maynas, departamento de Loreto.

\section{OBTENCIÓN DE LOS REPRODUCTORES}

Fueron seleccionados una hembra con presencia de óvulos maduros y cuatro machos maduros (emisión de semen por presión abdominal) de un total de 56 individuos adultos (del stock de reproductores identificados individualmente con microchips "Pit Tags"). De cada reproductor se colectó y conservó en alcohol al 96\% un fragmento de aleta anal para la extracción de DNA y posterior determinación del genotipo.

\section{OBTENCIÓN DE GENOTIPO DE REPRODUCTORES}

La extracción del DNA de los progenitores de doncella fue realizada mediante el método CTAB modificado (Doyle \& Doyle, 1987), a partir de la membrana Interradial de la aleta anal. Los genotipos fueron establecidos utilizando seis marcadores microsatélites diseñados para Pseudoplatystoma corruscans por Revaldaves et al., 2005 (Tabla 1). Las amplificaciones de PCR se llevaron a cabo en un volumen final de $10 \mu \mathrm{l}$, conteniendo $100 \mathrm{ng}$ de DNA en buffer PCR $1 \mathrm{X}, 0,4 \mu \mathrm{M}$ de cada primer, $0,2 \mathrm{mM}$ de dNTPs, 1,5 mM de $\mathrm{MgCl}, 0,4$ unidades de Taq polimerasa y agua 2 ultrapura. Las condiciones de amplificación fueron: 2 min a $92{ }^{\circ} \mathrm{C}$, seguido de 30 ciclos comprendidos por: 1 min a $95^{\circ} \mathrm{C}, 1 \mathrm{~min}$ a la temperatura de hibridación (Tabla 1 ) y 1 min a $72^{\circ} \mathrm{C}$, 
terminando con una extensión final de 10 min a 72 ${ }^{\circ} \mathrm{C}$. Los alelos fueron separados en un analizador genético Applied Biosystems 3130 para lo cual se preparó una solución que consiste en: $8.6 \mu \mathrm{l}$ de formamida, $0.4 \mu \mathrm{l}$ de ROX 500 y $1 \mu \mathrm{l}$ del producto de PCR. Los pesos de los alelos fueron determinados mediante el software Peak scannerversion1.0 (https://products.appliedbiosystems.com).

\section{OBTENCIÓN Y CULTIVO DE LA PROGENIE}

La reproducción inducida fue realizada según el protocolo de Nuñez et al. (2008). La fertilización fue realizada combinando $30 \mathrm{~g}$ de óvulos de una hembra (H) con una mezcla de esperma de cuatro machos (M). En total se obtuvieron cuatro familias (numeradas de 1 a 4) por la combinación de la hembra con los cuatro machos (numerados de 1 a 4). Una vez ocurrida la eclosión en las incubadoras, 3000 larvas a $1 \mathrm{dpf}$ (día post-fertilización) fueron colectadas al azar y transferidas a tres tanques de un sistema de circuito cerrado (1000 larvas por tanque), donde fueron mantenidos en completa oscuridad durante un periodo de 26 días para desarrollar varios experimentos (no descritos aquí) sobre su viabilidad, crecimiento y desarrollo ontogénico (Nuñez et al., 2011). La alimentación fue suministrada a partir del tercer día post-fertilización (dpf) y consistió en nauplios de artemia, con una frecuencia de cinco veces al día desde las 7 horas y 30 con un intervalo de 3 horas.

\section{MUESTREO DE LA PROGENIE}

Se realizaron tres muestreos al azar, con tres repeticiones y en las tres etapas del desarrollo de la progenie. El control 1 (Figura 1A) fue realizado a 1 dpf y consistió en una colecta de larvas. El control 2 (Figura 1B) fue realizado a los cinco días (alevines de $5 \mathrm{dpf}$ ). Finalmente el control 3 (Figura 1C) fue realizado a los 26 días (alevines de $26 \mathrm{dpf}$ ). Para cada control se colectaron al azar 50 individuos en cada una de las tres repeticiones. Cada uno de los individuos fue fotografiado (fotodocumentado), utilizando un microscopio estereoscopio Leica, y conservado en alcohol al 96\% hasta el momento del análisis del DNA.

\section{OBTENCIÓN DEL GENOTIPO DE LA PROGENIE}

El DNA de las larvas a $1 \mathrm{dpf}$ fue obtenido por trituración de todo el cuerpo del individuo; en los alevines a 5 dpf también se utilizó todo el cuerpo, a excepción del estómago por contener DNA exógeno (nauplios de artemia); mientras que en los alevines a 26 dpf sólo se utilizó la parte posterior del cuerpo. La extracción se realizó con el método CTAB (Doyle \& Doyle, 1987), realizándose una modificación al protocolo convencional, disminuyendo las proporciones de los reactivos a la mitad, debido al pequeño tamaño de las muestras. Las amplificacionesy los análisis de los alelos fueron realizados con el mismo protocolo utilizado en la obtención del genotipo de los reproductores.

Tabla 1. Locus, motivos de repetición, rango de tamaño y temperatura de hibridación de seis loci microsatélites utilizados para la asignación de parentesco en larvas de Pseudoplatystoma fasciatum.

\begin{tabular}{|c|c|c|c|c|c|}
\hline $\begin{array}{c}\text { LOCUS/ } \\
\mathrm{N}^{0} \text { DE } \\
\text { ACCESIONES }\end{array}$ & $\begin{array}{l}\text { MOTIVOS DE } \\
\text { REPETICIÓN }\end{array}$ & PRIMER $\left(5^{\prime} \rightarrow 3^{\prime}\right)$ & COLORANTE & $\begin{array}{c}\text { RANGO DE } \\
\text { TAMAÑO } \\
(\mathrm{pb})\end{array}$ & $\begin{array}{c}T \\
\left({ }^{\circ} \mathrm{C}\right)\end{array}$ \\
\hline $\begin{array}{l}\text { Pcor1 } \\
\text { AY737063 }\end{array}$ & $(\mathrm{TC})_{9} \mathrm{GC}(\mathrm{TC})_{9}$ & $\begin{array}{l}\text { F: AAACCCGAGGATAACCAGTC } \\
\text { R: CAGCGTGCTACTAACACAAAC }\end{array}$ & $\begin{array}{l}\text { FAM } \\
\text { ( azúl) }\end{array}$ & $104-120$ & 61.7 \\
\hline $\begin{array}{l}\text { Pcor2 } \\
\text { AY737064 }\end{array}$ & $(A G)_{19}$ & $\begin{array}{l}\text { F: GATATGCAAATAAGAAGGTC } \\
\text { R: TCTTCTGGCTTTTCCTCCTCT }\end{array}$ & $\begin{array}{c}\text { HEX } \\
\text { ( verde ) }\end{array}$ & $217-229$ & 58.3 \\
\hline $\begin{array}{l}\text { Pcor5 } \\
\text { AY737067 }\end{array}$ & $(\mathrm{TC})_{8} \mathrm{CC}(\mathrm{TC})_{15}$ & $\begin{array}{l}\text { F: GACTAAGATTACACAGAGATTC } \\
\text { R: CTTGGTGGGGAAACAGGC }\end{array}$ & $\begin{array}{c}\text { HEX } \\
\text { ( verde ) }\end{array}$ & $131-155$ & 59.1 \\
\hline $\begin{array}{l}\text { Pcor7 } \\
\text { AY737069 }\end{array}$ & $(\mathrm{AG})_{8} \mathrm{GG}(\mathrm{AG})_{2} \mathrm{CG}(\mathrm{AG})_{4}$ & $\begin{array}{l}\text { F: ATGCTGGGATACGCTCAGAC } \\
\text { R: GTGGCGAGTGAACAAGTCC }\end{array}$ & $\begin{array}{c}\text { NED } \\
\text { ( amarillo ) }\end{array}$ & $229-245$ & 65.5 \\
\hline $\begin{array}{l}\text { Pcor8 } \\
\text { AY737070 }\end{array}$ & $(\mathrm{AC}) 12$ & $\begin{array}{l}\text { F: ACACCATACGCACACACTCG } \\
\text { R: TGAGGTCGGGTGATAAGGTC }\end{array}$ & $\begin{array}{c}\text { HEX } \\
\text { ( verde ) }\end{array}$ & $167-183$ & 58.8 \\
\hline $\begin{array}{l}\text { Pcor10 } \\
\text { AY737072 }\end{array}$ & $(\mathrm{GTCG})_{15}(\mathrm{GT})_{9}$ & $\begin{array}{l}\text { F: TTTAAGACAGCACAGCCTGTGGGG } \\
\text { R: AAGACAGCGCCATAGAGTTCTGCC }\end{array}$ & $\begin{array}{l}\text { FAM } \\
\text { ( azúl ) }\end{array}$ & $146-156$ & 53.1 \\
\hline
\end{tabular}




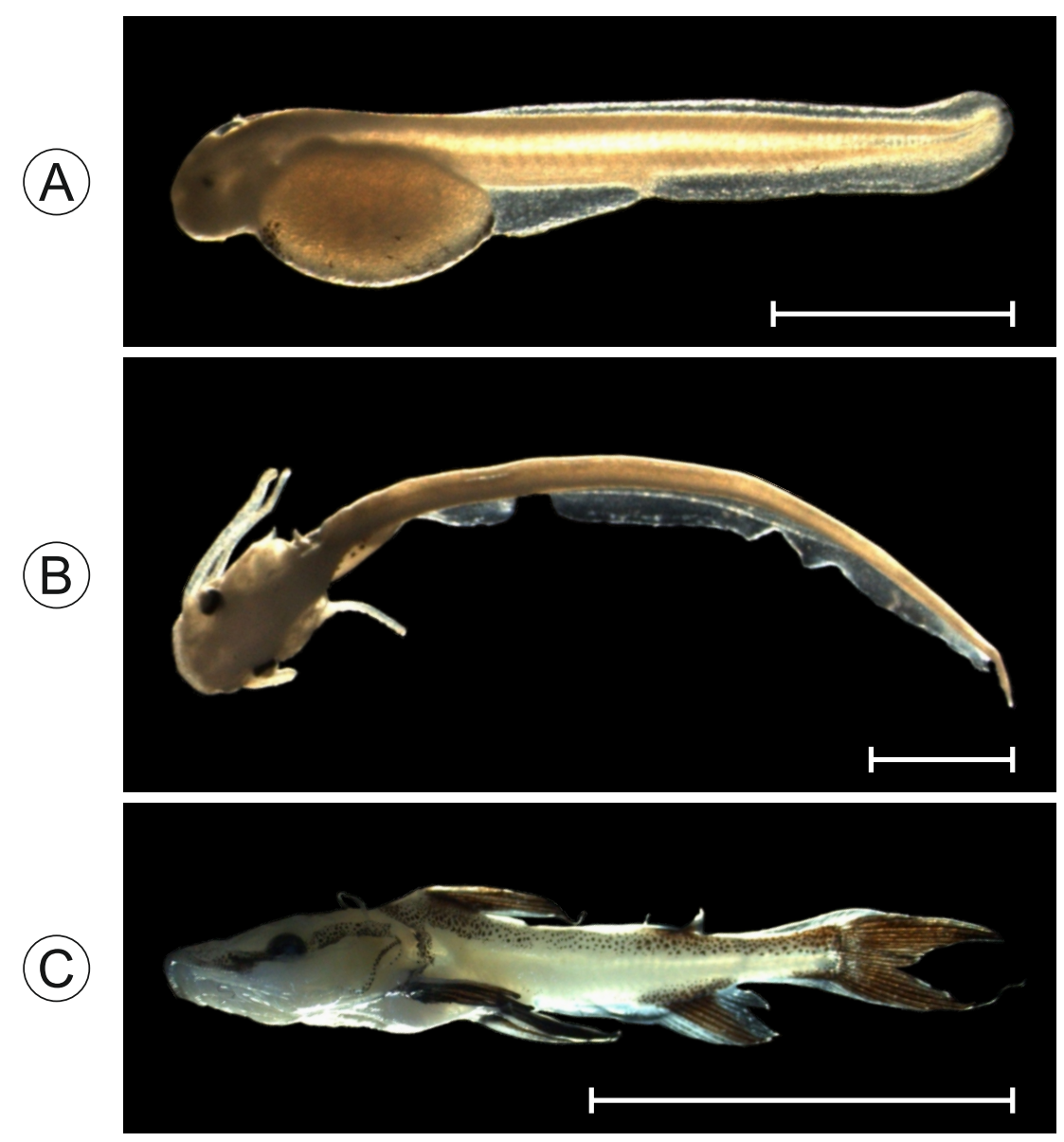

Figura 1. Vista lateral de la progenie de doncella Pseudoplatystoma fasciatum, (A) larva de $1 \mathrm{dpf}$, (B) alevín de $5 \mathrm{dpf}$, (C) alevín de $26 \mathrm{dpf}$. Escala Figuras A y B $(1 \mathrm{~mm})$; Figura C $(10 \mathrm{~mm})$.

\section{ASIGNACIÓN DE PARENTESCO}

Los perfiles de los microsatélites de los seis loci fueron utilizados para identificar a los progenitores de los 150 individuos analizados dentro de las cuatro familias.

El análisis fue realizado usando el software Probmax, (http://www.uoguelph.ca/ rdanzman/ software/PROBMAX/) el cual consiste en calcular la probabilidad de cada uno de los progenitores potenciales sea el padre del individuo considerado, con base en la compatibilidad entre los genotipos de ambos y teniendo conocimiento del genotipo de la madre.

Los resultados también fueron verificados manualmente buscando los alelos o combinaciones de alelos diagnósticos de las familias.

\section{RESULTADOS Y DISCUSIÓN \\ IDENTIFICACIÓN GENÉTICA DE LOS REPRODUCTORES}

De los seis loci microsatélites utilizados en este estudio, cinco, permitieron la diferenciación genética de los reproductores sin riesgo de equivocarse. El locus con mayor número de alelos diagnósticos entre los reproductores fue Pcor 8 (macho 2: alelos 167 y 169, macho 3: alelos 175 y 185, macho 1: alelos 181 y macho 4: alelo 179). En tanto que el locus Pcor 10 no presentó ninguno. En cuanto a los reproductores, el macho 4 presentó mayor número de alelos diagnósticos (8 alelos): Pcor 1 (104), (120), Pcor 2 (223), (225), Pcor 5 (155), Pcor 7 (229), (257) y Pcor 8 (179), en tanto que el macho 1 presentó solo dos: Pcor 5 (151) y Pcor 8 (181) (Tabla 2). 


\section{ASIGNACIÓN DE PARENTESCO DE LA PROGENIE}

En el muestreo 1 (Tabla 3) solo 3 larvas no presentaron alelo diagnóstico en sus genotipos, estableciéndose su parentesco con la familia 1 por la combinación diagnostica de alelos presente. En este control el mayor número de larvas perteneció a la familia 4 (23 larvas), y el menor número fue reportado para las familias 1 y 3 (5 larvas para cada una).

Los alevines de los muestreo 2 y 3 presentaron alelos diagnósticos en sus genotipos, permitiendo una fácil identificación de sus parentescos (Tabla 3). En el muestreo 2 el mayor número de alevines perteneció a la familia 2 (21 alevines) y el menor a la familia 1 (3 alevines). Mientras que en el muestreo 3, el mayor número de alevines perteneció a la familia 4 (23 alevines) y el menor a la familia 3 ( 5 alevines). No encontrándose ningún individuo de la familia 1.

\section{PERSPECTIVAS}

Actualmente los marcadores moleculares vienen siendo utilizados en la caracterización de stocks de reproductores, de su progenie y en programas de domesticación. Los marcadores aloenzimáticos han sido utilizados preferentemente hasta mediados de los años noventa en estudios de salmones (Pasdar et al., 1984; May \& Jonson, 1993), en peces del género Xiphophorus (Morizot et al., 1991), en el hirame Paralichthys olivaceus (Liu et al., 1997) entre otros.

Los marcadores moleculares RAPD fueron utilizados en bagres como el pez gato americano Ictalurus punctatus y el pez gato azul Ictalurus furcatus (Lui et al., 1998). En los últimos años la determinación de parentesco de peces como el lenguado japonés (Hara \& Sekino, 2003), el bacalao del atlántico Gadus morhua (Herlin et al., 2007), el lenguado senegalés Solea senegalensis (Castro et al., 2006), la Acedía del Japón Pleuronectes herzensteini (Kim et al., 2007), el rutilo Rutilus rutilus (Hamilton \& Tyler, 2008) y la anchoa europea Engraulis encrasicolus (Borrell et al., 2011) esta basada principalmente en marcadores microsatélites. La preferencia en el uso de este marcador es debido a su alto nivel de variación genética, es decir que son altamente polimórficos o informativos a nivel intrapoblacional (Liu \& Cordes, 2004). En cuanto que los marcadores isoenzimáticos presentan el problema de ausencia de polimorfismo para uno o varios de los loci disponibles para una especie, que son aproximadamente entre 10 a 20 por especie (Murphy et al., 1990), y los marcadores RAPD sufren de falta de reproducibilidad, ocasionando perfiles genéticos diferentes por variaciones de laboratorio a laboratorio o de diferentes termocicladores (Penner et al., 1993; Wang et al., 1993).

Antiguamente el monitoreo de las progenies de doncella podía ser realizado solo con las familias cultivadas separadamente. Esto dificultaba la evaluación de las respuestas de las progenies, pues no había certeza que la diferencias observadas se deba a diferencias intrínsecas de la progenie, o hayan sido inducidas por pequeñas variaciones medioambientales.

El estudio comunal solo era posible cuando los especímenes eran lo suficientemente grandes para que puedan ser diferenciados por marcación con pit tags.

La posibilidad de caracterizar los reproductores y asignar el parentesco a las progenies en diferentes momentos (etapas) durante el desarrollo de su historia de vida temprana, abre un horizonte nuevo a estos estudios permitiendo el monitoreo de parámetros en situación comunal con una alta pertinencia estadística, como por ejemplo el monitoreo de la velocidad de crecimiento, adaptación a dietas y resistencia, entre otros. Asimismo, evaluar en una forma más efectiva las diferencias entre las familias cultivadas en situación comunal.

Esto también ha sido demostrado en otras especies de peces tales como el salmón Atlántico (Salmo solar) y la trucha arco iris (Oncorhynchus mykiss) donde a partir de 8 y 4 marcadores microsatélites altamente polimórficos demostraron también que se puede determinar el parentesco en ausencia de marcadores físicos tags e información de pedigree a un grupo de $\mathrm{f}$ ami $\mathrm{l} i$ a s cul $\mathrm{t}$ ivada $\mathrm{s}$ en forma comuna 1, respectivamente (Norris et al., 2000; Herbinger et al., 1995).

Esta técnica también ha sido exitosamente usada en poblaciones naturales en el bacalao del Atlántico Gadus morhua donde identificaron el parentesco a 300 alevinos mediante 8 marcadores microsatélites, logrando evaluar la contribución parental del stock de reproductores mantenidos en cautiverio en estanques comerciales (Herlin et al., 2008). Así como en el rodaballo (Hippoglossus hipoglossus) donde mediante 5 marcadores microsatélites identificaron el parentesco a 145 alevines, logrando evaluar el impacto de selección de los individuos sobre la diversidad genética de la progenie F1 a ser utilizados en futuros stock de reproductores (Jackson et al., 2003). 
Tabla 2. Genotipos de los cinco reproductores de doncella Pseudoplatystoma fasciatum por cada locus analizado. Alelos diagnósticos para cada reproductor en negrita.

\begin{tabular}{|c|c|c|c|c|c|c|c|c|}
\hline \multirow{2}{*}{$\begin{array}{l}\text { REPRODUC } \\
\text { TORES }\end{array}$} & \multirow{2}{*}{ CóDIGO } & \multicolumn{6}{|c|}{$\mathrm{LOCI}$} & \multirow{2}{*}{$\begin{array}{c}\text { TOTAL DE } \\
\text { ALELOS } \\
\text { DIAGNÓSTICOS }\end{array}$} \\
\hline & & PCOR 1 & PCOR 2 & PCOR 5 & PCOR 7 & PCOR 8 & PCOR 10 & \\
\hline Hembra1 & 1E0D73 & 116118 & 223223 & 147149 & 245245 & 169169 & 146156 & 4 \\
\hline Macho 1 & 29309 & 112112 & 217221 & 145151 & 247247 & 181183 & 156156 & 2 \\
\hline Macho 2 & 23178 & 112112 & 221221 & 145149 & 239239 & 167169 & 156156 & 4 \\
\hline Macho 3 & 394572 & 110112 & 217229 & 131145 & 247247 & 175185 & 146156 & 4 \\
\hline Macho 4 & 111E1F & 104120 & 223225 & 131155 & 229257 & 179183 & 156156 & 8 \\
\hline $\begin{array}{r}\text { Total } \\
\text { diagnóst }\end{array}$ & $\begin{array}{l}\text { lelos } \\
\text { por loci }\end{array}$ & 5 & 3 & 4 & 4 & 6 & 0 & 22 \\
\hline
\end{tabular}

Tabla 3. Progenie de doncella Pseudoplatystoma fasciatum asignadas en cuatro familias.

\begin{tabular}{|c|c|c|c|c|c|}
\hline \multirow{2}{*}{ MUESTREOS } & \multicolumn{4}{|c|}{ FAMILIAS } & \multirow{2}{*}{ TOTAL } \\
\hline & FAMILIA 1 & FAMILIA 2 & FAMILIA 3 & FAMILIA 4 & \\
\hline Muestreo 1 & 5 & 17 & 5 & 23 & 50 \\
\hline Muestreo 2 & 3 & 21 & 7 & 19 & 50 \\
\hline Muestreo 3 & - & 22 & 5 & 23 & 50 \\
\hline Total de individuos analizados & 8 & 60 & 17 & 65 & 150 \\
\hline
\end{tabular}

\section{AGRADECIMIENTOS}

Al Proyecto Innovación y Competitividad para el Agro Peruano - INCAGRO, por el financiamiento parcial del presente estudio a través del subproyecto "Mejoramiento genético y producción intensiva de alevinos seleccionados de doncella Pseudoplatystoma fasciatum (Linnaeus, 1776) en la Amazonía peruana“.

\section{BIBLIOGRAFÍA CITADA}

Borrell, Y.; Álvarez, J.; Vásquez, E.; Sánchez, J.A.; Fernandez-Pato, C.; Martínez-Tapia, C.; Blanco, G. 2002. Aplicación de marcadores microsatélites a los programas de cultivo del roballo Scophthalmus maximus (L., 1758). Instituto Español de Oceanografía, 18 (1-4): 203-209.

Borrell, Y.J.; Álvarez, J.; Blanco G.; Martínez de Murguía, A.; Lee, D.; Fernández, C.; Martínez, C.; Cotano, U.; Alvarez, P.; Sanchez Prado, J.A. 2011. A parentage study using microsatellite loci in a pilot project for aquaculture of the European anchovy Engraulis encrasicolus L. Aquaculture, 310 (3-4): 305-311.

Castro, J.; Pino, A.; Herminda, M.; Bouza, C.; Riaza, A.; Ferreiro, I.; Sánchez, L.; Martínez, P. 2006. A microsatellite marker tool for parentage analysis in Senegal sole (Solea senegalensis): Genotyping errors, null alleles and conformance to theoretical assumptioms. Aquaculture, 261:1194-1203.

Chakraborty, R.; Meagher, T.; Smouse, P.E. 1988. Parentage analysis with genetic markers in natural populations I. The expected proportion of offspring with unambiguos paternity. Genetics, 118: 527-536.

DeWoody, J.A.; Avise, J.C. 2000. Microsatellite variation in marine, freshwater and anadromous fishes compared with other animals. Fish Biology, 56: 461-473.

Doyle, J.J.; Doyle, J.L. 1987. A rapid DNA isolation procedure for small quantities of fresh leaf tissue. Phytochemical Bulletin, 19: 11-15. 
Garber, A. F.; Tosh, J.J.; Fordham, S. E.; Hubert, S.; Simpson, G.; Symonds, J.E.; Robinson, J.A.B.; Bowman, S.; Trippel, E. A. 2010. Survival and growth traits at harvest of communally reared families of Atlantic cod (Gardus morhua). www.elservier.com/locate/equa-online.

García, A.; Tello, Salvador.; Vargas, G.; Duponchelle, F. 2009. Patterns of commercial fish landings in the Loreto region (Peruvian Amazon) between 1984 and 2006. Fish Physiology and Biochemestry, 35:53-67.

Goulding, M. 1980. The fishes and the forest: Explorations in Amazonian natural history. University of California Press, Berkeley, CA, USA. 280pp.

Hamilton, P.B.; Tyler, C.R. 2008. Identification of microsatellite loci for parentage 5804 analysis in roach Rutilus rutilus and 8 other cyprinid fish by cross-species 5805 amplification, and a novel test for detecting hybrids between roach and other 5806 cyprinids. Molecular Ecology Resources, 8: 462-465.

Hara, M.; Sekino, M. 2003. Efficient detection of parentage in a cultured Japanese flounder Paralichthys olivaceus using microsatellite DNA marker. Aquaculture, 217: 107-114.

Harris, A.S.; Bieger, S.; Doyle, R.W.; Wright, J.M. 1991. DNA fingerprinting of tilapia Oreochromis niloticus and its application to aquaculture genetics. Aquaculture, 92: 157-163. Herbinger, C.M.; Doyle, R.W.; Pitman, R.; Paquet, D.; Mesa, K.A.; Morris, B.; Wright, J.M.; Cook, D. 1995. DNA fingerprinting based analysis of paternal and maternal effects on offspring growth and survival in communally reared rainbow trout. Aquaculture, 137: 245256.

Herlin, M.; Delghandi, M.; Wesmajervi, M.; Taggart, J.B.; Mc Andrew, B. J.; Penman, D. J. 2008. Analysis of the parental contribution to a group of fry from a single day of spawning from a commercial Atlantic cod (Gadus morhua) breeding tank. Aquaculture, 274: 218-224.

Herlin, M.; Taggart, J.B.; McAndrew, B.J.; Penman, D.J. 2007. Parentage allocation in a complex situation: a large commercial Atlantic cod (Gadus morhua) mass spawning tank. Aquaculture, 272: 195-203.

Jackson, T. R.; Martín-Robichaud D.J.; Reith M.E. 2003. Application of DNA markers to the management of Atlantic halibut (Hipoglossus hippoglossus) broodstock. Aquaculture, 220: 245-259.

Kim, S.G.; Morishima, K.; Satoh, N.; Fujioka, T.; Saito, S.; Aran, K. 2007. Parentage assignment in hatchery population of brown sole Pleuronectes herzensteini by microsatellite DNA markers. Fisheries Science, 73 (5): 1087-1093.

Kellogg, K.A.; Markert, J.A.; Stauffer, J.R.; Kocher, T.D. 1995. Microsatellite Variation Demonstrates Multiple Paternity in Lekking Cichlid Fishes from Lake Malawi, Africa. The Royal Society, 260: 79-84.

Kossowski, C. 1996. Perspective de L'eìlevage dês poisons-chats (Siluroidei) en Ameìrique du Sud, Aquaticuatic Living Resources, 9: 189-195.

Liu, Z.J.; Cordes, J.F. 2004. DNA marker Technologies and their applications in aquaculture genetics. Aquaculture, 238: 1-37.

Liu, Z.J.; Li, P.; Argue, B.; Dunham, R. 1998. Inheritance of RAPD markers in channel catfish (Ictalurus punctatus), blue catfish (I. furcatus) and their F1, F2 and backcross hybrids. Animal Genetics, 29: 58-62.

Liu, S.; Ikeda, M.; Fujio, Y. 1997. Genetic features of natural and cultured populations in plaice (Paralichthys olivaceus).Tohoku Journal of Agricultural Research, 47: 85-96.

May, B.; Johnson, K.R. 1993. Composite linkage map of salmonid fishes (Salvelinus, Salmo, and Oncorhynchus). Genetic Maps: Locus Maps of Complex Genomes, (4) 309-317.

Montalvan, G. 2008. Ontogenia de la doncella, Pseudoplatystoma fasciatum (Linnaeus, 1766), y su relación con la dinámica del canibalismo. Tesis para optar el título de Ingeniero Agrónomo. Universidad Nacional Federico Villarreal, Facultad de Oceanografía, Pesquería y Ciencias Alimentarias, Escuela Profesional de Ingeniería en Acuicultura, Lima, Perú. 113pp.

Morizot, D.C.; Slaugenhaupt, S.A.; Kallman, K.D.: Chakravarti, A. 1991. Genetic linkage map of fishes of the genus Xiphophorus (Teleostei: Poeciliidae). Genetics, 127: 399-410.

Murphy, R.W.; Site, J.W.; Buth, D.J.; Haufler, C.H. 1990. Proteins I: Isoenzyme electrophoresis. In: Molecular Systematics. Hillis, D.M.; Moritz, C.(eds.). Sinauer Associates, Sunderland MA. 45-126pp.

Norris, A.T.; Bradley, D.G.; Cunningham, E.P. 2000. Parentage and relatedness determination in farmed Atlantic Salmon (Salmo solar) using microsatellite markers. Aquaculture, 182: 73-83.

Núñez, J.; Castro, D.; Fernández C.; Dugué R.; ChuKoo F.; Duponchelle F.; García C.; Renno, J.F. (2011) Hatching rate and larval growth variations in Pseudoplatystoma punctifer: maternal and paternal effects. Aquaculture Research, (en prensa) 
Nuñez, J. 2009. Domesticación de nouvelles espèces d'intérêt piscicole en Amazonie. Agricultures, 18:(2-3) 136-143.

Nuñez, J.; Dugué, R.; Arana, N.C.; Duponchelle, F.; Renno, J.F.; Raynaud, T.; Hubert, N.; Legendre, M., 2008: Induced breeding and larval rearing of Surubi, Pseudoplatystoma fasciatum (Linnaeus, 1766), from the Bolivian Amazon. Aquaculture Research, 39: 764-776.

Pasdar, M.; Philipp, D.P.; Whitt, G.S.1984. Linkage relationships of nine enzyme loci in sunfishes (Lepomis; Centrarchidae). Genetics 107: 435446.

Penner, G.A.; Bush, A.; Wisw, R.; Kim, W.; Domier, L.; Kasha, K.; Laroche, A.; Scoles, G.; Molnar, S.J.; Fedak, G. 1993. Reproducibility of random amplified polymorphic DNA (RAPD) analysis among laboratories. In: PCR methods and applications cold spring harbor laboratory press. 341-345 pp.

Pereira, L.H. G.; Foresti, F.; Oliveira, C. 2009. Genetic structure of the migratory catfish
Pseudoplatystoma corruscans (Siluriformes: Pimelodidae) suggests homing behavior. Ecology of Freshwater Fish 18: 215-225.

Reid, S. 1983. La biología de los bagres rayados Pseudoplatystoma fasciatum y P. tigrinum en la cuenca del río Apure, Venezuela. Revista UNELLEZ de Ciencia y Tecnología, 1: 13-41.

Revaldaves, E.; Pereira, L.; Foresti, F.; Oliveira, C. 2005. Isolation and characterization of microsatellite loci in Pseudoplatystoma corruscans (Siluriformes: Pimelodidae) and crossspecies amplification. Molecular ecology notes, 5: 463-465.

Tello, S.; García, A. 2009. La pesquería de grandes bagres en la región Loreto. Instituto de Investigaciones de la Amazonía Peruana. 28pp. Wang, D.; Waye, M.M.Y.; Taricani, M.; Buckingam,

K.; Sandham, H.J. 1993. Artifactual variation in randomly amplified polymorphic DNA banding patterns. Biotechniques 14:214-218. 